\title{
Stereotactic body radiotherapy vs radiofrequency ablation in the treatment of hepatocellular carcinoma: a meta-analysis
}

\section{Yang-Xun Pan}

Sun Yat-sen University Cancer Center

Qian Long

Sun Yat-sen University Cancer Center

\section{Dan-Dan Hu}

Sun Yat-sen University Cancer Center

\section{Yi-Zhen Fu}

Sun Yat-sen University Cancer Center Jun-Cheng Wang

Sun Yat-sen University Cancer Center

Mian Xi

Sun Yat-sen University Cancer Center

Shi-Liang Liu

Sun Yat-sen University Cancer Center

Li Xu

Sun Yat-sen University Cancer Center

Meng-Zhong Liu

Sun Yat-sen University Cancer Center

Min-Shan Chen

Sun Yat-sen University Cancer Center

Yao-Jun Zhang ( $\boldsymbol{\nabla}$ zhangyuj@sysucc.org.cn )

https://orcid.org/0000-0002-9752-4729

\section{Research article}

Keywords: radiofrequency ablation, stereotactic body radiotherapy, hepatocellular carcinoma, metaanalysis

Posted Date: September 19th, 2019

DOI: https://doi.org/10.21203/rs.2.14632/v1 
License: (c) (i) This work is licensed under a Creative Commons Attribution 4.0 International License. Read Full License

Version of Record: A version of this preprint was published at Frontiers in Oncology on October 29th, 2020. See the published version at https://doi.org/10.3389/fonc.2020.01639. 


\section{Abstract}

Background Both stereotactic body radiotherapy (SBRT) and radiofrequency ablation (RFA) are effective local treatments for hepatocellular carcinoma (HCC), but whether RFA is superior to SBRT is still controversial. Therefore, we performed a meta-analysis to compare the treatment outcomes of SBRT with RFA as curable or bridge intention.

Methods We searched online databases for studies that compared treatment outcome for SBRT and RFA. Eligibility criteria included evaluation of local control, overall survival (OS), transplant rate, and posttransplant pathological necrosis.

Results 10 retrospective studies with a total of 2732 patients were included. 2 studies were in favor of SBRT in local control, 2 studies preferred RFA in OS and others reported comparable outcomes for both. SBRT demonstrated significantly higher 1- and 3-year local control than RFA (OR $0.42,95 \% \mathrm{Cl} 0.24$ to $0.74, \mathrm{P}$ $=0.003 ; \mathrm{OR} 0.54,95 \% \mathrm{Cl} 0.37$ to $0.80, \mathrm{P}=0.002$, respectively). However, SBRT reported significantly shorter 1- and 2-year OS (OR 1.52, 95\% Cl 1.21 to 1.90, P =0.0003; OR 1.66, 95\% Cl 1.38 to 2.01, $\mathrm{P}<0.00001$, respectively). As bridge treatment, no significant difference was shown in transplant rate and posttransplant pathological necrosis rate $(\mathrm{OR} 0.57,95 \% \mathrm{Cl} 0.32$ to $1.03, \mathrm{P}=0.060 ; \mathrm{OR} 0.49,95 \% \mathrm{Cl} 0.13$ to 1.82 , $P=0.290$, respectively).

Conclusions This study demonstrates SBRT is able to complete a better local control for HCC than RFA, though the OS is inferior to RFA because of tumor burden or liver profiles of the enrolled studies. Welldesigned, randomized, multicenter trials will be required to further investigate the conclusion.

\section{Background}

Liver transplantation is the most beneficial therapy for early hepatocellular carcinoma (HCC) patients, as it removes both the tumor and the cirrhotic liver(1). However, given organ availability limitation, a number of patients that may benefit from this treatment have to stay on the waiting list for a long time. Therefore, stereotactic body radiotherapy (SBRT) and radiofrequency ablation (RFA) are offered as potentially alternative local control modalities for patients in the waiting list $(2,3)$.

RFA induces coagulative necrosis of tumor through thermal effect and is the first-line treatment for small $\mathrm{HCC}(\leq 3 \mathrm{~cm})$, providing comparable long-term outcome with resection(4). However, RFA has several contraindications, including large tumor size, lesions adjacent to major vessels or close to the liver hilum. As the above circumstances may result in incomplete ablation, which potentially leads to worse prognosis(5-9).

SBRT is an advanced technology that delivers ablative radiation doses to tumors in a few fractions while minimizing the dose to normal liver tissue. Early results with SBRT have shown considerable local control even for large tumors or HCC ineligible for surgery(10). Moreover, SBRT has been frequently used as an alternative to RFA for small HCC patients with tumors near critical anatomical structures or major vessels due to the heatsink effect that can occur with RFA(11). 
Recent publications have reported either comparable outcomes between the two treatment modalities or favorable outcomes for one to the other( $3,10,12-19)$. However, the efficacy of these two treatments regarding disease control and long-term survival are ununified. The aim of this meta-analysis is mainly to compare the benefits of SBRT and RFA in the local progression control and overall survival (OS) in the treatment for HCC.

\section{Methods}

\section{Study selection}

The inclusion criteria of this meta-analysis were: (1) diagnosed primary liver cancer definitively. Patients diagnosed with HCC based on pathological evidence from fine needle aspiration (FNA) or in the absence of biopsy evidence, based on imaging techniques including contrast-enhanced ultrasonography (CEUS), computed tomography (CT), and magnetic resonance imaging (MRI) companied with alpha-fetoprotein elevation; (2) no evidence of invasion into the major portal/hepatic vein branches or extrahepatic metastasis based on radiologic imagine; (3) patients without previous treatment of transcatheter arterial chemoembolization (TACE), surgery, chemotherapy, or other anti-tumor treatment; (4) documented indications for SBRT and RFA clearly; (5) either randomized controlled trials or retrospective studies were candidates; (6) patients of two groups with comparable basic clinical characters; (7) studies with outcome information regarding local progression (LP) rates, OS rates, and/or transplant rates.

Studies with following characteristics were excluded: (1) studies that did not report original data, including abstracts, case reports, expert opinions, editorials, reviews or letters; (2) either group in the studies combined other therapies; (3) studies based on the same cohort.

\section{Search strategy}

A systematic online databases search of PubMed Central, Embase, Cochrane Library and Google Scholar was separately conducted by two reviewers to identify all relevant availability of studies until August $26^{\text {th }}$ 2019.

This meta-analysis was performed in consistent with the Preferred Reporting In Systematic Reviews and Meta-Analysis (PRISMA) statement checklist. The subject headings (MeSH) search and text word search were used, including "radiofrequency ablation", "stereotactic body radiation therapy" and "hepatocellular carcinoma". These terms were used in different combinations. Only human beings and English language studies were included. A manual research was performed by browsing all references of all identified studies. This progressed research was repeated to ensure to include the whole relevant studies. The research was completed by two reviewers before the data analysis independently (Y-X. Pan and M. X). If a study was controversial, the corresponding author was asked to judge (Y-J. Zhang).

\section{Data collection}


Data were extracted from the included studies, including: number of patients in the SBRT and RFA groups, age, gender, primary tumor size, number of tumors, median dose of SBRT, Child-Pugh class, median followup time, 1-, 2- and 3-LP rates, 1-, 2-, 3- and 5-year OS, post-transplant necrosis rate and time to liver transplant.

\section{Definitions}

Local progression was defined as the recurrence of lesion in the treatment area by imaging studies. And LP time was the period from the initial treatment to the discovery of LP or last follow-up. The OS was defined the period from the date of initial treatment of the HCC to the date of death related to any cause. Transplant rate was the proportion of patients who received liver transplantation after SBRT or RFA therapies. Posttransplant pathological necrosis was evaluated by post-transplant pathology.

\section{Statistical analysis and synthesis}

All analyses were performed with the help of statistical software, named Review Manager, version 5.3 (Nordic Cochrane Centre; Oxford, England). For data evaluation, patients were assinged into two groups: the SBRT-treated group and the RFA-treated group. The odds ratio (OR) and/or hazard ratios (HR) accompanying $95 \%$ confidence intervals $(95 \% \mathrm{Cl})$ were calculated for dichotomous and univariable analysis outcomes in terms of LP, OS and prognostic factor on treatment allocation, respectively.

Meanwhile, we assessed the heterogeneity among trials according to the Chi-squared $\left(\chi^{2}\right)$ test including the inconsistency factor $\left(1^{2}\right)$. The heterogeneity was defined as a $P$ value of less than 0.05 or an $I^{2}$ greater than $40 \%$. A random-effect model was applied when the heterogeneity $\left(I^{2}<40 \%\right)$ was absent or low, or we use a fixed-effect model if the heterogeneity $\left(I^{2}>40 \%\right)$ was moderate or high(20). A potential publication bias was assessed by visually inspecting the Begg's funnel plots in which the standard error (SE) of log OR or log HR was plotted against the OR or HR, respectively.

\section{Results}

\section{Search results and quality assessment}

A total of 440 studies were identified for the first time from PubMed by the search strategy previously established and 269 studies were identified via other sources or review. Subsequently, 11 studies were deleted for duplication with the help of Mendeley (Elsevier Inc.; Atlanta, GA, USA). The titles and abstracts of 270 studies were then screened for inclusion. The full texts of 36 studies were read and, finally, we included 10 non-randomized controlled trial (Non-RCT) studies that met the present meta-analysis criteria $(3,10,13-16,18,19,21,22)$. The details of PRISMA flow diagram of the literature for meta-analysis is shown in Supplementary Figure S1(23).

In present analysis, 3 studies were based on already existed database $(13,15,19)$ and the remaining 7 studies were based on retrospective studies(3,10,16-18,21,22). 2 studies conducted SBRT or RFA for 
transplant intent. 5 studies were performed in USA(10,13,15,18,19), 2 in Japan(12,16), 1 in Canada(3), 1 in South Korea(17), and 1 in China(22). Out of 2732 patients from the 10 included studies 859 patients were classified into the SBRT group, and the rest 1873 patients were classified into the RFA group. The Newcastle Ottawa Scale (NOS)(24) were used to assess the quality of non-randomized studies. Among all studies included, over $50 \%$ of which were medium-score studies because of inconsistent comparability, although the sections of selection and outcomes were relatively appropriate qualities. Therefore, we believed that the present meta-analysis possesses a medium-class of quality (Supplementary Table S1).

Among included studies, 2 studies were in favor of SBRT on local control(10,21), 2 studies preferred to RFA on OS $(15,19)$ and others reported comparable outcomes between groups(3,13,16-18,22). Notably, according to baseline characteristics, several studies enrolled patients in SBRT group were prone to suffer from larger tumor diameter $(3,10,16,19)$ and higher proportion of Child-Pugh C patients(18) (Table 1). These groups were evaluated for therapeutic efficacy in treating HCC patients. The details of the studies included in the present meta-analysis are listed in Table 1. 
Table 1

Characteristics of

include studies

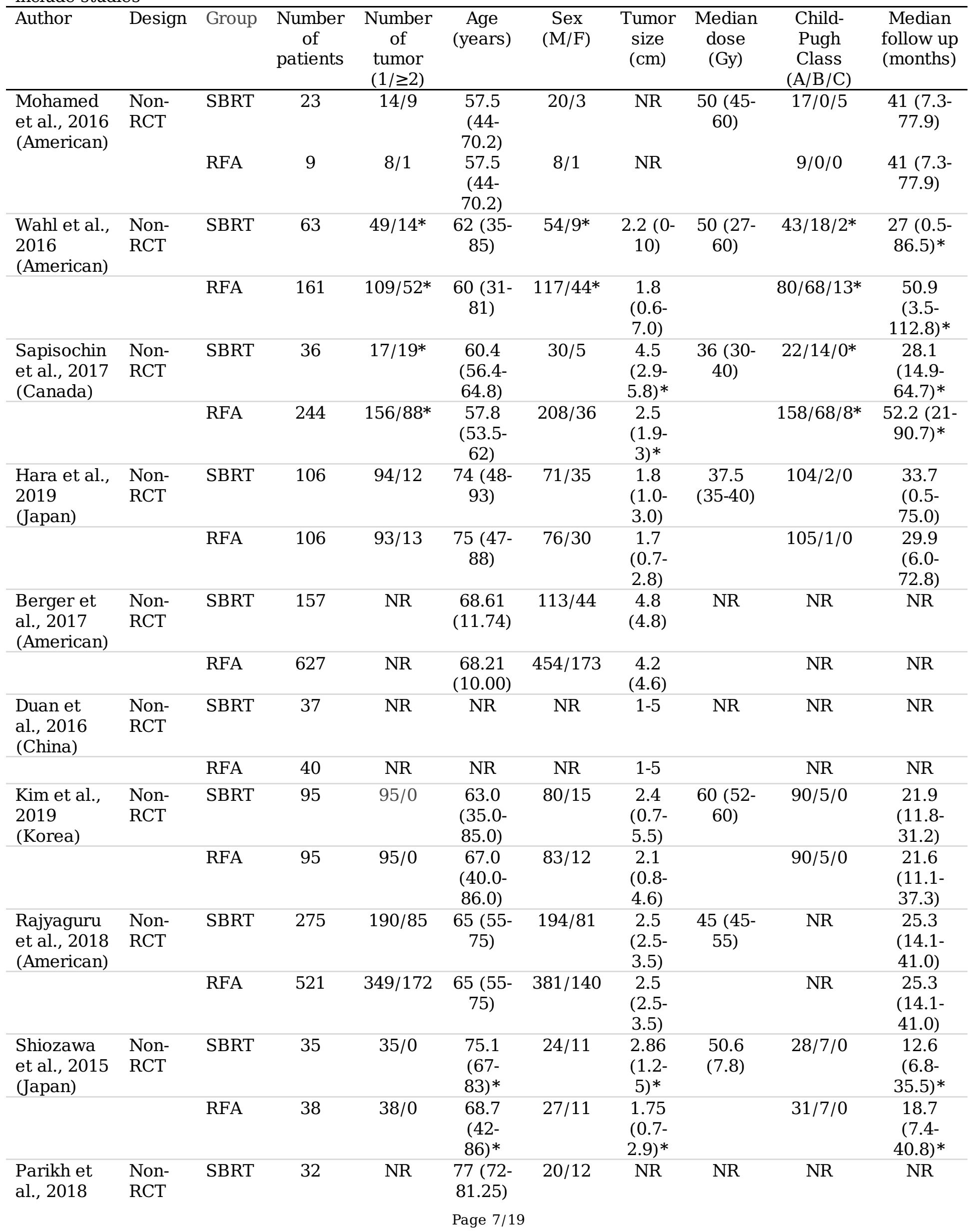


(American)

$\begin{array}{llllllll}\text { RFA } & 32 & \text { NR } & 79(76- & 22 / 10 & \text { NR } & \text { NR } & \text { NR }\end{array}$

$\begin{array}{llr}\text { Total } & \text { SBRT } & 859 \\ & \text { RFA } & 1873\end{array}$

SBRT, stereotactic body radiotherapy; RFA, radiofrequency ablation; NR, not reported. *Statistically significant $(P<0.05)$.

\section{Local progression rates}

6 out of the 10 studies illustrated 1-, 2- and 3-year LP rates(10,16-18,21,22). Our pooled results showed that the SBRT group had significantly better 1- and 3-year local control rates than the RFA group (OR 0.42 , $95 \% \mathrm{Cl} 0.24$ to $0.74, P=0.003$; OR $0.54,95 \% \mathrm{Cl} 0.37$ to $0.80, P=0.002$, respectively). However, the 2-year LP rate showed marginal benefit of SBRT group than RFA group (OR $0.67,95 \% \mathrm{Cl} 0.43$ to $1.05, P=0.080)$. No heterogeneity was shown among the studies of $1-, 2$ - and 3-year local progression rates $\left(X^{2}=2.93,1^{2}=0 \%\right.$; $\chi^{2}=0.33, I^{2}=0 \% ; \chi^{2}=2.66, I^{2}=0 \%$, respectively), so the fixed effect model was used to pool the outcomes (Figure 1).

\section{Overall Survival}

9 studies with 2700 patients compared OS rates of SBRT group with RFA group $(3,10,13,15-17,19,21,22), 1$ study with 280 patients were excluded as both SBRT and RFA were applied as bridge therapies before transplantation and thus the actual OS rates of SBRT or RFA might be affected by subsequent transplantation(3). Heterogeneity was low for the studies that reported 1 - and 2-year OS rates $\left(X^{2}=10.47, I^{2}\right.$ $=33 \% ; \chi^{2}=7.93,1^{2}=24 \%$, respectively), so the fixed effect model was used to pool the outcomes. However, high heterogeneities were observed in the studies that reported 3 - and 5 -year OS rates $\left(\chi^{2}=20.54,1^{2}=76 \%\right.$; $\chi^{2}=12.88, I^{2}=77 \%$, respectively), so the random effect model was used to pool the outcomes (Figure 2 ). The pool results showed that 1- and 2-year OS rates of RFA group were better than SBRT group (OR 1.52, $95 \% \mathrm{Cl} 1.21$ to $1.90, P=0.0003 ; \mathrm{OR} 1.66,95 \% \mathrm{Cl} 1.38$ to $2.01, P<0.00001$, respectively). Whereas, there were no differences for 3 - and 5-year OS rates in both groups (OR 1.44, 95\% $\mathrm{Cl} 0.90$ to 2.33, $P=0.130$; OR 1.35, $95 \% \mathrm{Cl} 0.81$ to $2.26, P=0.250$, respectively).

Additionally, a secondary analysis was performed to control the potential report bias, and we enrolled the studies that reported outcomes of both LP and OS. As result, the 1-, 2-, 3- and 5-year OS rates indicated no significant difference between both groups (OR $0.96,95 \% \mathrm{Cl} 0.59$ to $1.57, P=0.870$; OR $1.35,95 \% \mathrm{Cl} 0.89$ to $2.03, P=0.160 ;$ OR $0.97,95 \% \mathrm{Cl} 0.28$ to $3.36, P=0.960 ; \mathrm{OR} 0.65,95 \% \mathrm{Cl} 0.37$ to $1.16, P=0.150$, respectively) (Supplementary Figure S2).

\section{Prognosis for treatment allocation}

3 and 5 studies evaluated the results of treatment allocation as a prognostic factor for $\operatorname{LP}(10,16,17)$ and OS $(13,15,17,19,21)$, respectively. The treatment allocation was not a prognostic factor for LP [HR $0.80,95 \%$ 
IC 0.58 to $1.09, P=0.160$ ]. However, RFA group was more favorable than SBRT group for OS benefits (HR $1.43,95 \%$ IC 1.23 to $1.80, P<0.00001)$. Heterogeneity did not exist among the studies $\left(\chi^{2}=5.39, I^{2}=63 \% ; \chi^{2}\right.$ $=3.64, I^{2}=0 \%$, respectively), so the fixed effect model was applied to pool the outcomes (Figure 3 ).

\section{Transplant and post-transplant pathological necrosis rate}

3 and 2 studies reported the transplant rate and post-transplant pathological necrosis rate, respectively $(3,10,18)$. There were no significant differences in transplant rate and post-transplant pathological necrosis rate for both SBRT and RFA (OR $0.57,95 \% \mathrm{Cl} 0.32$ to $1.03, P=0.060 ; \mathrm{OR} 0.49,95 \% \mathrm{Cl}$ 0.13 to $1.82, P=0.290$, respectively). No significant heterogeneity was observed when the transplant and post-transplant pathological necrosis rates were evaluated $\left(\chi^{2}=4.55, I^{2}=56 \% ; \chi^{2}=1.03, I^{2}=3 \%\right.$, respectively) and the fixed-effect model was used (Figure 4).

\section{Publication bias}

The Begg's funnel plot was used to evaluate the reliability of publication bias in our meta-analysis(25). The shape of 11 funnel plots was basically inverted and bilateral symmetry. Therefore, these results indicated that there was little publication bias among all comparisons in this meta-analysis (Supplementary Figure S3).

\section{Discussion}

The main finding of this meta-analysis is that SBRT showed a better local control than RFA for patients with HCC, though the 1- and 2-year OS rates of SBRT were inferior because of the tumor burden and liver profiles. Recently, RFA, as a traditional curable treatment, was challenged by $\operatorname{SBRT}(15,21,22)$. With the development of imaging technique, such as four-dimensional computed tomography (4DCT), SBRT is able to provide a more precise picture of HCC for treatment design(26). This improvement of SBRT effectively fixed the deficiencies of the high incomplete ablation rate of RFA under several specific conditions(7).

In 2006, Romero et al. firstly applied the radiational technique of SBRT as a salvage treatment to control the primary and metastatic liver tumors(27). Although only 25 patients with 45 lesions that were unfit for other local control treatment were included in this study, the results indicated that SBRT was feasible with acceptable toxicity and local control efficacy. They also pointed out that for patients with Child B accompanied by high toxicity risks, the optimal dose-fractionation schemes had to be found. Inspired by Romero et al.,n other investigators mainly focused on the application of SBRT on unresectable HCC or its effect in combination with other therapies(28-30). All these studies proved that SBRT was safe and provided satisfying local control for HCC, which encouraged the temptation of expanding the indications of SBRT from a salvage or bridge treatment to curable intention.

RFA has long been applied as the first-line treatment for small HCC according to several clinical practice guidelines, including European Society for Medical Oncology (ESMO) and American Association for the 
Study of Liver Disease (AASLD)(31,32). However, RFA still suffers from high incidence of local incomplete ablation because of technical limitations (varies from $2 \%$ to $60 \%(5-9)$ ) and it required additional or combination therapies(33). As an advanced technique that shows reliable local control on HCC, SBRT have been considered as a potentially alternative therapy to RFA.

Many observational or retrospective studies in recent years have indicated that SBRT showed a non-inferior local control rate compared to RFA(10,16-18,22). Shiozawa et al. first compared the clinical outcomes between SBRT and RFA for HCC patients in a pilot study in 2015 and reported that SBRT was likely to become an important option for local treatment of early $\operatorname{HCC}(16)$. The subsequent studies indicated that SBRT appeared to be a reasonable alternative treatment of inoperable HCC in 2016(10,22). Subsequently, several large volume validation studies based on online database had also proved comparable outcomes between SBRT and RFA regarding local control(13,19). Moreover, in 2019, Kim et al. retrospectively reviewed the institutional database for RFA and SBRT with curative intent, and they revealed that SBRT appears to be an effective alternative treatment for HCC when RFA is not feasible due to tumor location or size(17). However, another database validation from the American national cancer database revealed better OS of RFA than SBRT(15). A meta-analysis is needed to attain definitive proof to solve these debates. Thus, we performed this meta-analysis to help identify the advantages and disadvantages of SBRT and RFA in HCC.

In the present meta-analysis, with respect to local control, SBRT showed significantly lower 1-and 3-year LP rates, which indicated that SBRT achieved superior local control to RFA in treating HCC. In detail, there were 5 and 4 studies enrolled the 1- and 3-year LP rate analysis, respectively. Among these studies, studies from Wahl et al. and Duan et al. were based on patients with inoperable $\operatorname{HCC}(10,22)$, studies of Hara et al. and Shiozawa et al. were based on patients with early-stage $\operatorname{HCC}(16,21)$ and studies from Kim et al. and Mohamed et al. did not specify patients characteristics $(17,18)$. Most of the enrolled studies tended to draw the conclusions with respect to local control that supported SBRT. Moreover, Wahl et al. and Hara et al. reached significantly favorable results for SBRT in treating inoperable and early-stage HCC, respectively $(10,21)$. However, only Kim et al. clarified the information of HCC location(17), others did not refer this critical factor which might influence the local control of RFA in our previous study(34). Additionally, treatment allocation was not a significance prognostic factor based on prognostic analysis. Therefore, further studies are needed to guarantee the appropriate individual treatment allocation.

Although, SBRT enjoyed higher local control rates than RFA in the present study, the 1- and 2-year OS rates of SBRT were significantly lower than RFA. Notably, Berger et al.((19) and Rajyaguru et al.(15) who reported favorable OS rates of RFA with large sample volume did not illustrate the LP rates correspondingly. There were two main reasons to the contradictory results between OS rates and LP rates. Firstly, there might be report bias when analyzing LP rates for both groups which might result in inconsistent outcomes between LP rates and OS rates because of unreported LP. Therefore, we conducted the secondary analysis on OS rates that included the studies that reported both LP rates and OS rates, and found that the 1-, 2-, 3- and 5year OS rates were comparable between SBRT and RFA. Secondly, as the first-line treatment, RFA is more likely to be assigned to patients with better conditions. Patients underwent SBRT were prone to suffer from larger tumor size and worse liver function, which indicated worse prognosis and decreased OS. Interestingly, the 3- and 5-year OS rates showed no significance in both groups, indicating that patients who 
did not die of tumor burden or liver functions in a short time might finally benefit from both treatments similarly. Meanwhile, RFA showed significant survival benefit for prognostic analysis on treatment allocation, which also could be explained by the reasons above. Therefore, we believed that the real effects of SBRT and RFA on long-term survival need to be further validated by high-level evidence, including randomized clinical trial (RCT).

As for bridge therapies to transplant, both SBRT and RFA provided a similar effect on patients waiting for transplantation. And the post-transplant pathological necrosis rate was comparable between both groups. These outcomes indicated that SBRT can be safely utilized as a bridge treatment to patients in the waiting list for transplantation with HCC, as an alternative to conventional bridging therapies. However, only 3 studies and 536 patients in total compared these parameters and more solid studies were needed to be enrolled in future.

This meta-analysis suffered from several limitations. First of all, only retrospective studies were available, resulting in relatively low quality of the evidence for the whole pooled results. Secondly, there are a total of 10 studies with 2732 participators, but the sample sizes of 4 studies were relatively small. A further sensitivity analysis on the factors affecting outcomes could not be applied. Therefore, some well-designed, large, prospective, and multi-center studies are desperately needed to obtain more solid evidence.

\section{Conclusion}

In sum, our meta-analysis shows that SBRT provided better local control than RFA, and it could be used as a potentially alternative local control treatment for HCC.

\section{Abbreviations}

SBRT = stereotactic body radiotherapy

RFA $=$ radiofrequency ablation

$\mathrm{HCC}=$ hepatocellular carcinoma

OS = overall survival

FNA $=$ fine needle aspiration

CEUS $=$ contrast-enhanced ultrasonography

$\mathrm{CT}$ = computed tomography

$\mathrm{MRI}=$ magnetic resonance imaging

TACE $=$ transcatheter arterial chemoembolization

PRISMA = Preferred Reporting In Systematic Reviews and Meta-Analysis 
$\mathrm{OR}=$ odds ratio

$\mathrm{HR}=$ hazard ratios

$\mathrm{SE}=$ standard error

Non-RCT $=$ non-randomized controlled trial

NOS $=$ Newcastle Ottawa Scale

4DCT $=$ four-dimensional computed tomography

$E S M O=$ European Society for Medical Oncology

AASLD = American Association for the Study of Liver Disease

\section{Declarations}

\section{Ethics approval and consent to participate}

Not applicable

\section{Consent for publication}

Not applicable.

\section{Availability of data and materials}

The datasets used and/or analysed during the current study are available from the corresponding author on reasonable request.

\section{Competing interests}

The authors declare that they have no competing interests.

\section{Funding}

This work was supported by the National Key R\&D Program of China (2018ZX10723204;

2018ZX10302205).

\section{Authors' contributions}


YXP, QL and DDH designed experiments and drafted the manuscript. YZF, JCW and MX were responsible for data collection and statistical analysis. SLL, LX and MZL helped revise the manuscript. MSC and YJZ approved the final version.

\section{Acknowledgments}

The authors acknowledge and express their deepest gratitude to the participants of this study. This manuscript has been revised by native English speaker for effective communication to a professional medical audience.

\section{References}

1. Sapisochin G and Bruix J: Liver transplantation for hepatocellular carcinoma: outcomes and novel surgical approaches. Nat Rev Gastroenterol Hepatol 14: 203-217, 2017.

2. Panel NHC: Hepatobiliary Cancers, V.1.2010. Nccn Guidel 1: 1-142, 2019.

3. G. S, A. B, M. D, et al.: Stereotactic body radiotherapy vs. TACE or RFA as a bridge to transplant in patients with hepatocellular carcinoma. An intention-to-treat analysis. J Hepatol 67: 92-99, 2017.

4. Chen M-S, Li J-Q, Zheng Y, et al.: A prospective randomized trial comparing percutaneous local ablative therapy and partial hepatectomy for small hepatocellular carcinoma. Ann Surg 243: 321-328, 2006.

5. N'Kontchou G, Mahamoudi A, Aout M, et al.: Radiofrequency ablation of hepatocellular carcinoma: long-term results and prognostic factors in 235 Western patients with cirrhosis. Hepatology 50: 14751483, 2009.

6. Tateishi R, Shiina S, Teratani T, et al.: Percutaneous radiofrequency ablation for hepatocellular carcinoma. An analysis of 1000 cases. Cancer 103: 1201-1209, 2005.

7. Waki K, Aikata H, Katamura Y, et al.: Percutaneous radiofrequency ablation as first-line treatment for small hepatocellular carcinoma: results and prognostic factors on long-term follow up. J Gastroenterol Hepatol 25: 597-604, 2010.

8. Granata V, Petrillo M, Fusco R, et al.: Surveillance of HCC Patients after Liver RFA: Role of MRI with Hepatospecific Contrast versus Three-Phase CT Scan-Experience of High Volume Oncologic Institute. Gastroenterol Res Pract 2013: 469097, 2013.

9. Doyle A, Gorgen A, Muaddi H, et al.: Outcomes of Radiofrequency Ablation as First-Line Therapy for Hepatocellular Carcinoma less than $3 \mathrm{~cm}$ in Potentially Transplantable Patients. J Hepatol, 2019.

10. Wahl DR, Stenmark MH, Tao Y, et al.: Outcomes after stereotactic body radiotherapy or radiofrequency ablation for hepatocellular carcinoma. J Clin Oncol 34: 452-459, 2016.

11. Feng M, Suresh K, Schipper MJ, et al.: Individualized Adaptive Stereotactic Body Radiotherapy for Liver Tumors in Patients at High Risk for Liver Damage: A Phase 2 Clinical Trial. JAMA Oncol 4: 40-47, 2018.

12. K. H, A. T, Y. T, et al.: Clinical outcomes after treatment for hepatocellular carcinoma, stereotactic body radiotherapy vs radiofrequency ablation: A propensity score-matched analysis. Hepatology 68: 848A, 
2018.

13. Parikh ND, Marshall VD, Green M, et al.: Effectiveness and cost of radiofrequency ablation and stereotactic body radiotherapy for treatment of early-stage hepatocellular carcinoma: An analysis of SEER-medicare. J Med Imaging Radiat Oncol 62: 673-681, 2018.

14. D. R. W, M. H. S, E. P, et al.: SBRT provides equivalent local control compared to RFA for the treatment of hepatocellular carcinoma with minimal toxicity. Int J Radiat Oncol Biol Phys 90: S378-S379, 2014.

15. D. J. R, A. J. B, A. L. S, et al.: Radiofrequency ablation versus stereotactic body radiotherapy for localized hepatocellular carcinoma in nonsurgically managed patients: Analysis of the national cancer database. J Clin Oncol 36: 600-608, 2018.

16. K. S, M. W, T. I, et al.: Comparison of percutaneous radiofrequency ablation and CyberKnife ${ }^{\circledR}$ for initial solitary hepatocellular carcinoma: A pilot study. World J Gastroenterol 21: 13490-13499, 2015.

17. Kim N, Kim HJ, Won JY, et al.: Retrospective analysis of stereotactic body radiation therapy efficacy over radiofrequency ablation for hepatocellular carcinoma. Radiother Oncol 131: 81-87, 2019.

18. M. M, A. W. K, M. A. T, et al.: Comparison of outcomes between SBRT, yttrium-90 radioembolization, transarterial chemoembolization, and radiofrequency ablation as bridge to transplant for hepatocellular carcinoma. Adv Radiat Oncol 1: 35-42, 2016.

19. Berger NG, Tanious MN, Hammad AY, et al.: External radiation or ablation for solitary hepatocellular carcinoma: A survival analysis of the SEER database. J Surg Oncol 116: 307-312, 2017.

20. Melsen WG, Bootsma MCJ, Rovers MM and Bonten MJM: The effects of clinical and statistical heterogeneity on the predictive values of results from meta-analyses. Clin Microbiol Infect 20: 123129, 2014.

21. Hara K, Takeda A, Tsurugai Y, et al.: Radiotherapy for Hepatocellular Carcinoma Results in Comparable Survival to Radiofrequency Ablation: a Propensity Score Analysis. Hepatology, 2019.

22. X. D, T. Z, H. X, J. S, W. H and H. X: Stereotactic body radiotherapy vs. radiofrequency ablation in the Treatment of small hepatocellular Carcinoma. Hepatology 64: 653A-654A, 2016.

23. Tian J, Zhang J, Ge L, Yang K and Song F: The methodological and reporting quality of systematic reviews from China and the USA are similar. J Clin Epidemiol 85: 50-58, 2017.

24. Stang A: Critical evaluation of the Newcastle-Ottawa scale for the assessment of the quality of nonrandomized studies in meta-analyses. Eur J Epidemiol 25: 603-605, 2010.

25. Higgins JPT, Altman DG, Gotzsche PC, et al.: The Cochrane Collaboration's tool for assessing risk of bias in randomised trials. BMJ 343: d5928, 2011.

26. Couri T and Pillai A: Goals and targets for personalized therapy for HCC. Hepatol Int 13: 125-137, 2019.

27. A.M. R, W. W, S. M. H, et al.: Stereotactic body radiation therapy for primary and metastatic liver tumors: A single institution phase i-ii study. Acta Oncol (Madr) 45: 831-837, 2006.

28. J. S, K. G, D. E, et al.: Cyberknife stereotactic body radiation therapy for nonresectable tumors of the liver: Preliminary Results. HPB Surg 2010, 2010. 
29. S. H. B, J. H. K, J. W. J, et al.: Stereotactic body radiation therapy using Cyberknife for primary hepatocellular carcinoma ineligible for local ablation therapy or surgical resection. Hepatology 50 : 1100A, 2009.

30. B. O. C, I. B. C, H. S. J, et al.: Stereotactic body radiation therapy with or without transarterial chemoembolization for patients with primary hepatocellular carcinoma: Preliminary analysis. BMC Cancer 8, 2008.

31. Heimbach JK, Kulik LM, Finn RS, et al.: AASLD guidelines for the treatment of hepatocellular carcinoma. Hepatology 67: 358-380, 2018.

32. Vogel A, Cervantes A, Chau I, et al.: Hepatocellular carcinoma: ESMO Clinical Practice Guidelines for diagnosis, treatment and follow-upt. Ann Oncol 29: iv238-iv255, 2018.

33. Peng Z-W, Zhang Y-J, Chen M-S, et al.: Radiofrequency ablation with or without transcatheter arterial chemoembolization in the treatment of hepatocellular carcinoma: a prospective randomized trial. $J$ Clin Oncol 31: 426-432, 2013.

34. Chen J, Peng K, Hu D, et al.: Tumor location influences oncologic outcomes of hepatocellular carcinoma patients undergoing radiofrequency ablation. Cancers (Basel) 10: 1-14, 2018.

\section{Figures}




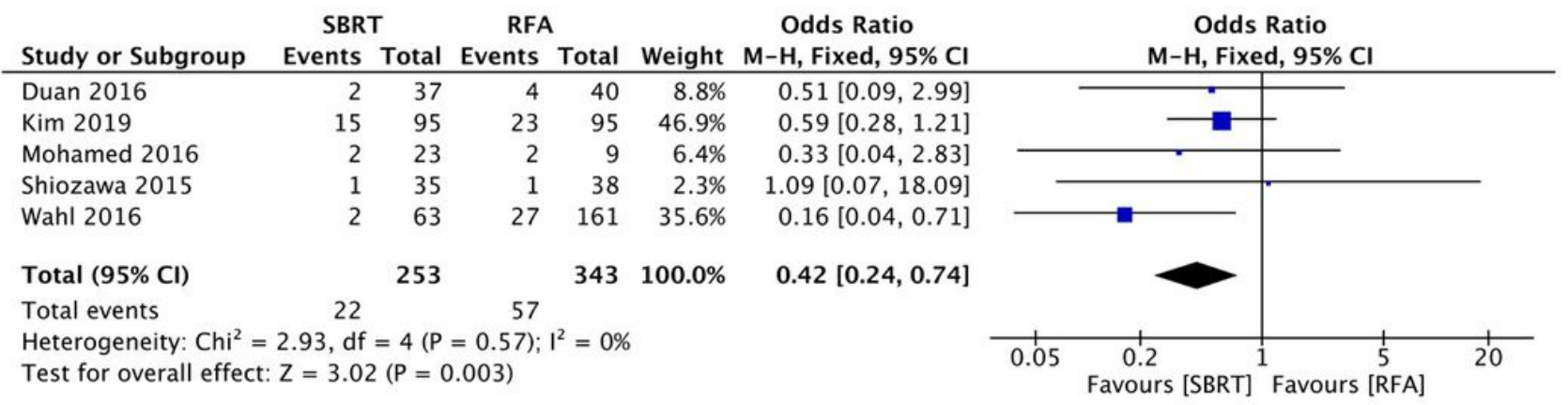

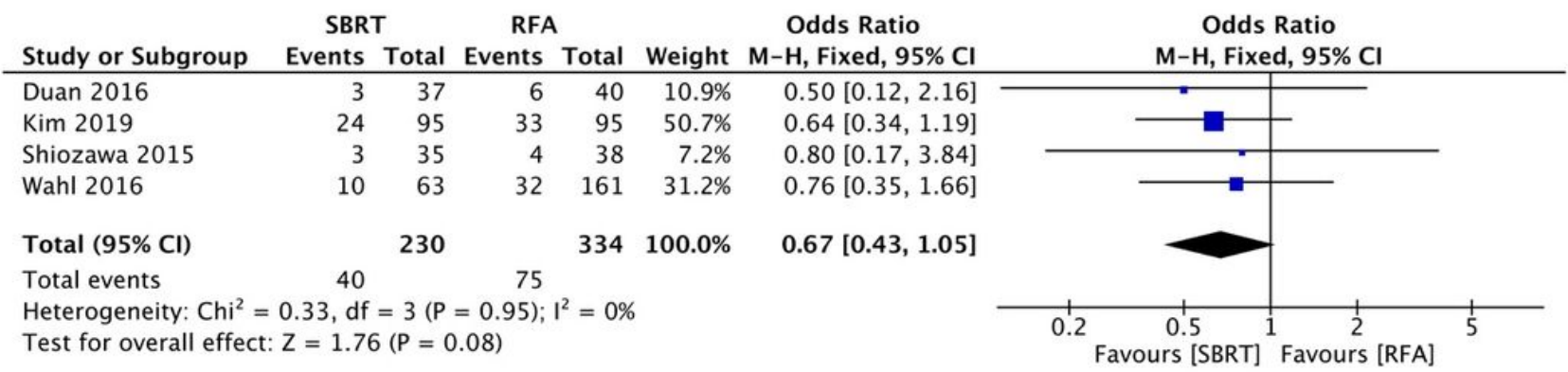

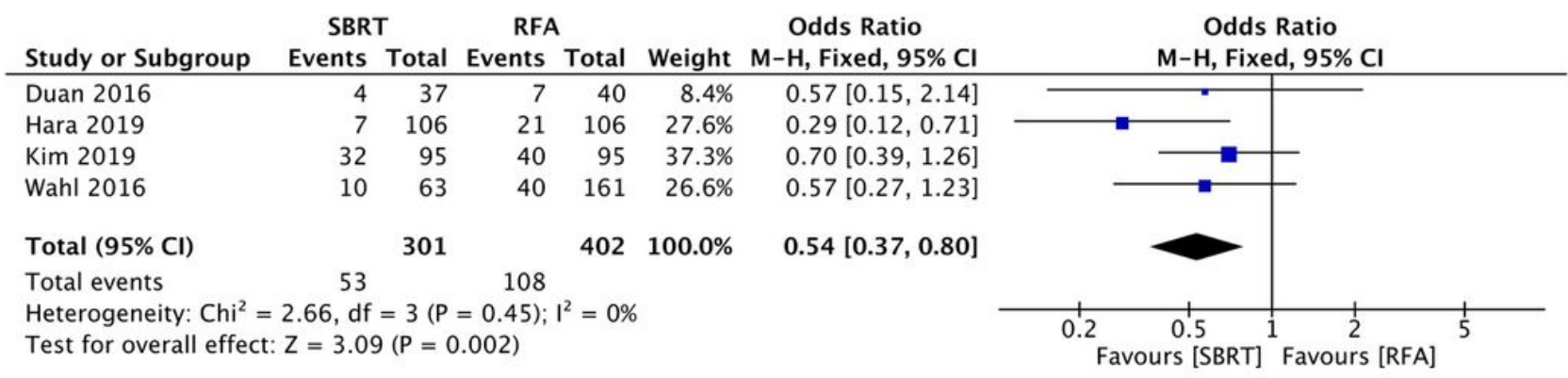

\section{Figure 1}

Forest plots demonstrating 1-, 2- and 3-years LP in SBRT and RFA for HCC 


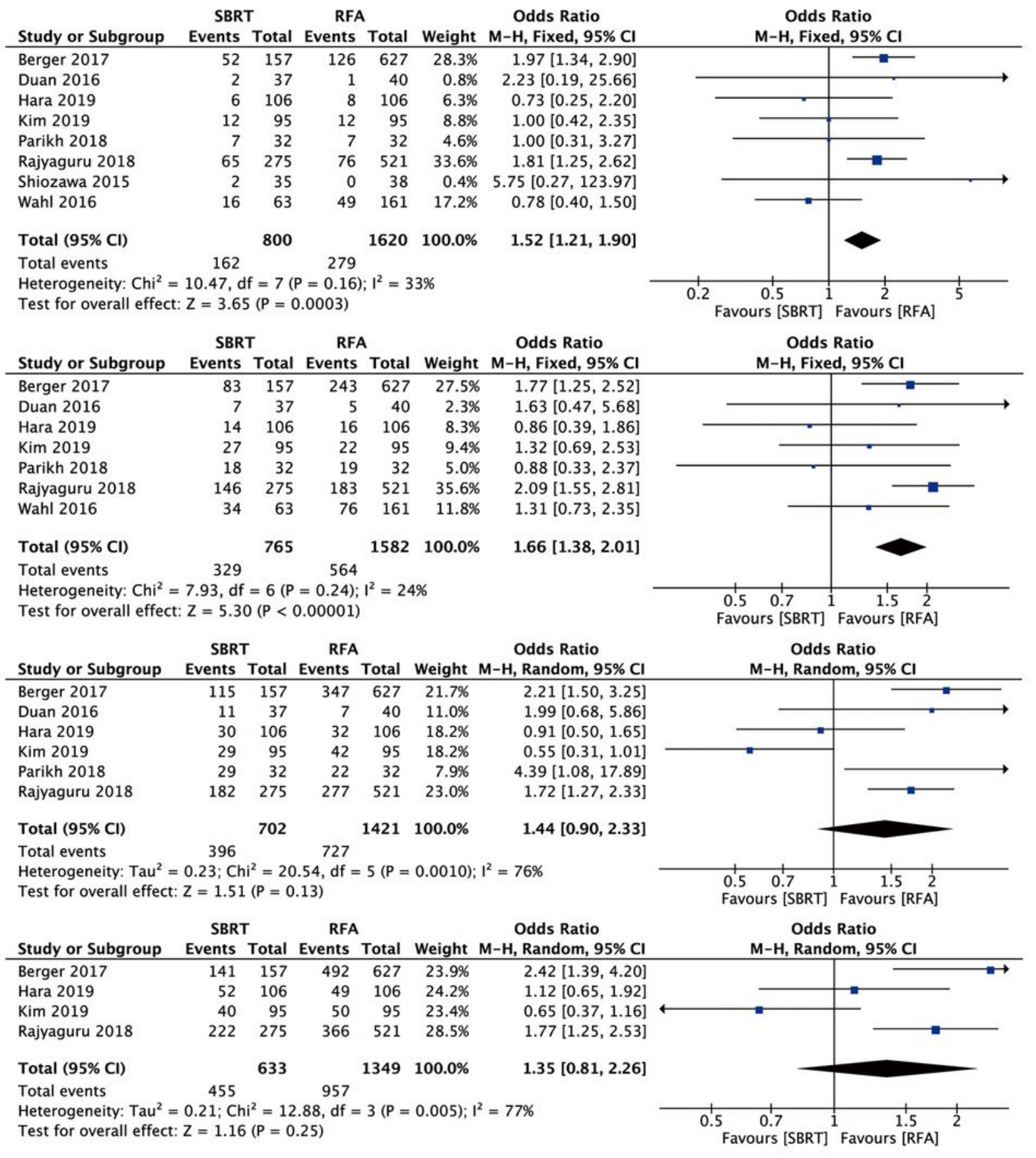

\section{Figure 2}

Forest plots demonstrating 1-, 2-, 3- and 5-years LP in SBRT and RFA for HCC 


\begin{tabular}{|c|c|c|c|c|c|c|}
\hline Study or Subgroup & log[Hazard Ratio] & SE & Weight & $\begin{array}{l}\text { Hazard Ratio } \\
\text { IV, Fixed, } 95 \% \mathrm{CI}\end{array}$ & \multicolumn{2}{|c|}{$\begin{array}{c}\text { Hazard Ratio } \\
\text { IV, Fixed, } 95 \% \mathrm{CI}\end{array}$} \\
\hline Kim 2019 & -0.3216 & 0.2785 & $34.1 \%$ & $0.72[0.42,1.25]$ & 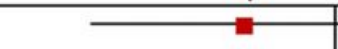 & \\
\hline Shiozawa 2015 & 0.0862 & 0.2318 & $49.2 \%$ & $1.09[0.69,1.72]$ & & \\
\hline Wahl 2016 & -0.9676 & 0.3985 & $16.7 \%$ & $0.38[0.17,0.83]$ & $\longleftrightarrow$ & \\
\hline Total $(95 \% \mathrm{Cl})$ & & & $100.0 \%$ & $0.80[0.58,1.09]$ & & \\
\hline \multicolumn{5}{|c|}{$\begin{array}{l}\text { Heterogeneity: } \mathrm{Chi}^{2}=5.39, \mathrm{df}=2(P=0.07) ; \mathrm{I}^{2}=63 \% \\
\text { Test for overall effect: } \mathrm{Z}=1.40(P=0.16)\end{array}$} & $\begin{array}{ll}0.5 & 0.7 \\
\text { Favours } & {[\mathrm{SBRT}]}\end{array}$ & 1 Favours [RFA] \\
\hline Study or Subgroup & log[Hazard Ratio] & SE & Weight & $\begin{array}{l}\text { Hazard Ratio } \\
\text { IV, Fixed, } 95 \% \mathrm{CI}\end{array}$ & \multicolumn{2}{|c|}{$\begin{array}{l}\text { Hazard Ratio } \\
\text { IV, Fixed, } 95 \% \mathrm{Cl}\end{array}$} \\
\hline Berger 2017 & 0.4383 & 0.1263 & $31.7 \%$ & $1.55[1.21,1.99]$ & & \\
\hline Hara 2019 & -0.0101 & 0.2555 & $7.8 \%$ & $0.99[0.60,1.63]$ & & \\
\hline Kim 2019 & 0.0862 & 0.3132 & $5.2 \%$ & $1.09[0.59,2.01]$ & & \\
\hline Parikh 2018 & 0.1398 & 0.4565 & $2.4 \%$ & $1.15[0.47,2.81]$ & & \\
\hline Rajyaguru 2018 & 0.3988 & 0.0978 & $52.9 \%$ & $1.49[1.23,1.80]$ & & \\
\hline Total $(95 \% \mathrm{CI})$ & & & $100.0 \%$ & $1.43[1.24,1.64]$ & & \\
\hline \multicolumn{5}{|c|}{$\begin{array}{l}\text { Heterogeneity: } \text { Chi }^{2}=3.64, \mathrm{df}=4(P=0.46) ; I^{2}=0 \% \\
\text { Test for overall effect: } Z=5.02(P<0.00001)\end{array}$} & $\begin{array}{cc}1 & 1 \\
0.5 & 0.7 \\
\text { Favours } & {[\mathrm{SBRT}]}\end{array}$ & $1 \frac{1}{1} \quad 1.5 \quad 2$ \\
\hline
\end{tabular}

\section{Figure 3}

Forest plots demonstrating prognostic factor for LP and OS regarding to treatment allocation.

\begin{tabular}{|c|c|c|c|c|c|}
\hline \multirow[b]{2}{*}{ Study or Subgroup } & \multicolumn{2}{|c|}{ SBRT } & \multicolumn{2}{|c|}{ RFA } & \multirow[b]{2}{*}{ Weight } \\
\hline & Events & Total & Events & Total & \\
\hline Mohamed 2016 & 14 & 23 & 5 & 9 & $9.6 \%$ \\
\hline Sapisochin 2017 & 30 & 36 & 203 & 244 & $29.6 \%$ \\
\hline Wahl 2016 & 4 & 63 & 34 & 161 & $60.9 \%$ \\
\hline Total $(95 \% \mathrm{Cl})$ & & 122 & & 414 & $100.0 \%$ \\
\hline Total events & 48 & & 242 & & \\
\hline
\end{tabular}

Odds Ratio

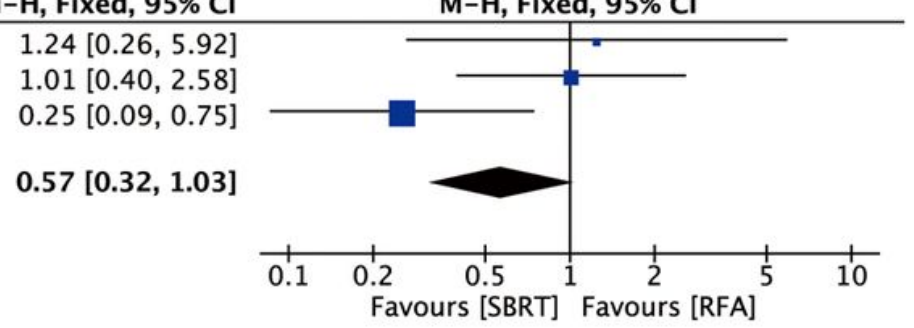

$\begin{array}{lll}\text { SBRT RFA } & \text { Odds Ratio }\end{array}$

\begin{tabular}{|c|c|c|c|c|c|c|c|c|c|}
\hline Study or Subgroup & Events & Total & Events & Total & Weight & M-H, Fixed, $95 \% \mathrm{Cl}$ & M-H, Fixe & d, $95 \% \mathrm{Cl}$ & \\
\hline Mohamed 2016 & 12 & 14 & 4 & 5 & $14.3 \%$ & $1.50[0.11,21.31]$ & & 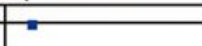 & \\
\hline Sapisochin 2017 & 27 & 30 & 196 & 203 & $85.7 \%$ & $0.32[0.08,1.32]$ & & - & \\
\hline Total $(95 \% \mathrm{Cl})$ & & 44 & & 208 & $100.0 \%$ & $0.49[0.13,1.82]$ & & & \\
\hline Total events & 39 & & 200 & & & & & & \\
\hline $\begin{array}{l}\text { Heterogeneity: } \mathrm{Chi}^{2} \\
\text { Test for overall effec }\end{array}$ & $\begin{array}{l}1.03, \mathrm{df} \\
Z=1.07\end{array}$ & $\begin{array}{l}=1(\mathrm{P} \\
7(\mathrm{P}=0\end{array}$ & $\begin{array}{l}=0.31) \\
0.29)\end{array}$ & $1^{2}=3 \%$ & & & $\begin{array}{c}0.1 \\
\text { Favours [SBRT] }\end{array}$ & \begin{tabular}{|cc} 
& 10 \\
Favours [RFA]
\end{tabular} & 100 \\
\hline
\end{tabular}

\section{Figure 4}

Forest plot demonstrating transplant rate and post-transplant pathologic necrosis in SBRT and RFA for HCC. 


\section{Supplementary Files}

This is a list of supplementary files associated with this preprint. Click to download.

- SupplementaryFigureS01V2.0.jpg

- SupplementaryFigureS03Ver2.0.jpg

- SupplementaryFigureS02Ver2.0.jpg

- SupplementaryTableS1.docx 